\title{
Contrasting Epidemiology and Clinicopathology of Female Breast Cancer in Asians vs the US Population
}

\author{
Ching-Hung Lin, Yoon Sim Yap, Kyung-Hun Lee, Seock-Ah Im, Yoichi Naito, Winnie Yeo, \\ Takayuki Ueno, Ava Kwong, Huiping Li, Shu-Min Huang, Roland Leung, Wonshik Han, \\ Benita Tan, Fu-Chang Hu, Chiun-Sheng Huang, Ann-Lii Cheng, Yen-Shen Lu; and The \\ Asian Breast Cancer Cooperative Group
}

See the Notes section for the full list of authors' affiliations.

Correspondence to: Yen-Shen Lu, MD, PhD, Department of Oncology, National Taiwan University Hospital, 7 Chung-Shan South Road, Taipei 10016, Taiwan (e-mail: yslu@ntu.edu.tw).

\begin{abstract}
Background: The incidence of breast cancer among younger East Asian women has been increasing rapidly over recent decades. This international collaborative study systemically compared the differences in age-specific incidences and pathological characteristics of breast cancer in East Asian women and women of predominantly European ancestry.

Methods: We excerpted analytic data from six national cancer registries (979 675 cases) and eight hospitals (18 008 cases) in East Asian countries and/or regions and, for comparisons, from the US Surveillance, Epidemiology, and End Results program database. Linear regression analyses of age-specific incidences of female breast cancer and logistic regression analyses of age-specific pathological characteristics of breast cancer were performed. All statistical tests were two-sided.

Results: Unlike female colorectal cancer, the age-specific incidences of breast cancer among East Asian women aged 59 years and younger increased disproportionally over recent decades relative to rates in US contemporaries. For years 2010-2014, the estimated age-specific probability of estrogen receptor positivity increased with age in American patients, whereas that of triple-negative breast cancer (TNBC) declined with age. No similar trends were evident in East Asian patients; their probability of estrogen receptor positivity at age 40-49 years was statistically significantly higher (odd ratio [OR] $=1.50,95 \%$ confidence interval $[\mathrm{CI}]=1.36$ to $1.67, \mathrm{P}<.001)$ and of TNBC was statistically significantly lower $(\mathrm{OR}=0.79,95 \% \mathrm{CI}=0.71$ to $0.88, \mathrm{P}<.001)$, whereas the probability of ER positivity at age 50-59years was statistically significantly lower (OR $=0.88,95 \% \mathrm{CI}=0.828$ to $0.95, P<.001)$. Subgroup analyses of US Surveillance, Epidemiology, and End Results data showed similarly distinct patterns between East Asian American and white American patients.

Conclusions: Contrasting age-specific incidences and pathological characteristics of breast cancer between East Asian and American women, as well as between East Asian Americans and white Americans, suggests racial differences in the biology.
\end{abstract}

Although Asian women have lower incidence rates of invasive breast cancer than Western women, the incidence of female breast cancer in East and Southeast Asia has increased rapidly over the past 40 years (1-3). Previous age-period-cohort analyses showed a strong cohort effect on female breast cancer incidence in Asia $(4,5)$, which has resulted in notable differences in age-specific incidences of breast cancer between Asian and
Western females. Specifically, the incidence of breast cancer peaked at around age 50 years in Hong Kong, Japan, Korea, and Taiwan compared to approximately 70 years in the United States $(2,3,6,7)$. Incidence rates of breast cancer among younger women in some East Asian populations have even surpassed that of recent generations in the United States (8). 
This strong cohort effect suggests that lifestyle and environmental changes might be major influences on the pathogenesis of breast cancer in Asian women. Rapidly increasing breast cancer incidence in East Asia has primarily been attributed to "Westernization" including: 1) dietary factors (eg, high fat intake, low vegetable consumption, and low soybean intake) and 2) reproductive factors (eg, delayed childbearing, low parity, less breastfeeding, early menarche, and late menopause) $(9,10)$. If rising breast cancer incidence rates were simply due to Westernization, emerging age-specific patterns in modern Asian women would eventually resemble those of Western populations. Therefore, a recent study showed longitudinal agespecific incidence rates with converging incidence rate ratios and concluded that the age effects for invasive breast cancer were more similar among Asian and Western populations than might be expected according to the extrapolated estimates (8). However, some evidence suggests racial effects on breast carcinogenesis and tumor biology in younger women. For example, overweight and/or obesity were inversely associated with premenopausal breast cancer risk in Europids and African Americans, but positively correlated in Asian women (11,12). A meta-analysis of breast cancer risk found a protective effect of soybean consumption in Asian and Asian American women, but not in Europids (13). Additionally, 13 genetic polymorphisms associated with breast cancer in Europeans failed to discriminate breast cancer risk in Singaporean Chinese, Malays, or Indians (14). Recently, multi-omics profiling of breast cancers in younger Korean patients has revealed distinctive molecular signatures, suggesting a more immune-active tumor microenvironment compared with that of Western counterparts (15).

Given the highly debatable issue about the similarity or dissimilarity in epidemiology and tumor biology of young female breast cancer between East Asia and Western countries, we conducted this multiregion study and explored the underlying pathophysiologic factors. We systematically compared temporal trends in age-specific incidences and pathological characteristics of female breast cancer among East Asians, Asian Americans, and non-Asian Americans. Female colorectal cancer was used as a control for the effect of Westernized lifestyle $(16,17)$.

\section{Methods}

\section{Registries and Databases Used for Data Collection}

The incidence rates of female breast cancer and female colorectal cancer in 5-year intervals for the years of 1973-2014 were obtained from the National Cancer Center of Japan (18), the Korea Central Cancer Registry (19), the National Registry of Disease Office of Singapore (20), and the Taiwan Cancer Registry (21) (Table 1). The age-specific incidence data for China and Hong Kong were excerpted from the International Agency for Research on Cancer database (22), but only data from Shanghai were retrieved for China because the other Chinese registries covered shorter time periods. For comparisons with the United States, age-specific incidence data were retrieved from the US Surveillance, Epidemiology, and End Results (SEER) database (23). Age-specific incidence rates of female breast cancer were estimated from 979675 cases in East Asians and 645633 in Americans. The time frames of age-specific incidence data varied according to the years available in national registries; for example, the female breast cancer incidence rates of Japan were not available until 2003. This study was approved by the institutional review boards of the National Taiwan University Hospital (201510027RINB).

To investigate the age-specific pathological characteristics of female breast cancer, hospitals in Beijing, Hong Kong, Japan, South Korea, Singapore, and Taiwan provided records of breast cancers diagnosed during years 2010-2014 (Table 1). For comparisons with the United States, age-specific pathological features of breast cancer among women diagnosed during 2010-2013 were ascertained from the SEER database (24). Age-specific clinicopathological characteristics of female breast cancer were determined based on 18008 cases in East Asians and 244819 in Americans. Positivity for estrogen receptor (ER+), progesterone receptor $(\mathrm{PR}+$ ), and human epidermal growth factor receptor 2 (HER2+) were defined according to the criteria of the individual hospitals. For data specifying proportions of ER and PR expression, we defined $1 \%$ as the threshold for ER+, based on the American Society of Clinical Oncology and College of American Pathologists guideline (25), and $10 \%$ as the cutoff for PR+, because past studies showed that a higher $\mathrm{PR}+$ threshold has better prognostic value $(26,27)$. Either ER+ or PR+ was defined as hormone receptor positive $(\mathrm{HR}+)$. Tumors with negative ER, PR, and HER2 staining were defined as triple-negative breast cancer (TNBC).

Using the SEER 18 database (24), we compared the agespecific pathological characteristics of female breast cancer from years 2010 to 2013 among Chinese, Japanese, and Korean Americans (C-J-K Asian Americans) and white Americans. The corresponding age-specific incidences of female breast and colorectal cancer from years 1990 to 2010 were estimated from the SEER 11 database (28).

\section{Statistical Analysis}

We plotted age-specific incidence rates of female breast and colorectal cancers in 5-year intervals for years 1973-2014 for China (Shanghai), Hong Kong, Japan, South Korea, Singapore, Taiwan, and the United States (SEER data). We performed multivariate linear regression analyses of age-specific incidence rates of female breast and colorectal cancers, averaged for years 20112014, using data from all national registries, except China (Shanghai) and Hong Kong, because of insufficient data (Table 1). We then plotted predicted age-specific incidence rates against age-groups to examine their congruence with corresponding observed values for each country and/or region. The time frame of 2011-2014 was chosen to reflect the contemporary impact of Westernized lifestyle.

Next, we used data from participating hospitals in East Asia and US SEER data to plot observed age-specific pathological characteristics of female breast cancer, including the proportions of ER+, PR+, HER2+, HR+/HER2-, HR-/HER2-, HR+/HER2+, HR-/HER2+, and histological grade III subtypes. We then fitted multiple logistic regression models for grouped binomial data including the proportions of ER+, PR+, HER2+, HR+/HER2-, HR-/ HER2-, HR+/HER2+, HR-/HER2+, and histological grade III subtypes of female breast cancer with data pooled for years 20102014. We also charted conditional-effect plots of predicted agespecific probabilities of ER+, PR+, HER2+, HR+/HER2-, HR-/ HER2-, HR+/HER2+, HR-/HER2+, and histological grade III breast cancer subtypes in 10-year intervals for East Asia and the United States to examine their congruence with corresponding observed age-specific positive proportions.

Finally, we plotted the age-specific proportions of ER+, PR+, HER2+, HR+/HER2-, HR-/HER2-, HR+/HER2+, HR-/HER2+, and histological grade III subtypes in 10-year intervals for years 
Table 1. National and hospital data sources, sample sizes, and survey periods

\begin{tabular}{|c|c|c|c|c|c|}
\hline \multirow[b]{2}{*}{ Country and/or Region } & \multicolumn{2}{|c|}{ National } & \multicolumn{3}{|c|}{ Hospital } \\
\hline & Data source & Years covered & Data source & Sample & Years covered \\
\hline China & $\begin{array}{l}\text { International Agency for } \\
\text { Research on Cancer }\end{array}$ & Shanghai: 1988-2007 & Beijing Cancer Hospital & 316 & 2010-2012 \\
\hline \multirow[t]{2}{*}{ Hong Kong } & $\begin{array}{l}\text { International Agency for } \\
\text { Research on Cancer }\end{array}$ & $1983-2007$ & $\begin{array}{l}\text { Prince of Wales Hospital/ } \\
\text { Chinese University of Hong } \\
\text { Kong }\end{array}$ & 630 & 2012 \\
\hline & & & $\begin{array}{l}\text { University of Hong Kong, Hong } \\
\text { Kong West Cluster Database }\end{array}$ & 1655 & 2010-2014 \\
\hline \multirow[t]{2}{*}{ Japan } & National Cancer Center & Breast cancer: 2003-2012 & Kyorin University Hospital & 487 & 2010-2012 \\
\hline & & $\begin{array}{l}\text { Colorectal cancer: } \\
\text { 1975-2012 }\end{array}$ & $\begin{array}{l}\text { National Cancer Center } \\
\text { Hospital East }\end{array}$ & 1464 & 2010-2014 \\
\hline Korea & $\begin{array}{l}\text { Korea Central Cancer } \\
\text { Registry }\end{array}$ & $2002-2013$ & $\begin{array}{l}\text { Seoul National University } \\
\text { Hospital }\end{array}$ & 5216 & 2010-2014 \\
\hline Singapore & $\begin{array}{l}\text { National Registry of } \\
\text { Diseases Office }\end{array}$ & 1980-2014 & $\begin{array}{l}\text { Singapore General Hospital/ } \\
\text { National Cancer Centre } \\
\text { Singapore }\end{array}$ & 4832 & 2010-2014 \\
\hline Taiwan & Taiwan Cancer Registry & 1979-2013 & $\begin{array}{l}\text { National Taiwan University } \\
\text { Hospital }\end{array}$ & 3408 & 2010-2014 \\
\hline United States & $\begin{array}{l}\text { National Cancer Institute, } \\
\text { Surveillance, } \\
\text { Epidemiology, and End } \\
\text { Results (SEER) Program }\end{array}$ & $\begin{array}{l}\text { SEER 9: 1973-2013 } \\
\text { SEER 11: 1990-2010 }\end{array}$ & $\begin{array}{l}\text { National Cancer Institute, SEER } \\
\text { Program }\end{array}$ & 244819 & SEER 18:2010-2013 \\
\hline
\end{tabular}

2010-2013 against age groups for C-J-K Asian Americans and white Americans, with pointwise two-sample $z$ tests to verify racial differences in the age-specific pathological characteristics of female breast cancer.

A two-sided $P$ value of no more than .05 was considered statistically significant. Statistical analysis was performed using R 3.3.1 software (R Foundation for Statistical Computing, Vienna, Austria).

\section{Results}

Trends in Age-Specific Incidence of Breast Cancer Between the United States and East Asia and Between non-Hispanic White Americans and Asian Americans

Supplementary Figure 1 (available online) shows the age-specific incidence rates of female breast cancer and female colorectal cancer over years 1973-2014 in the United States and East Asian countries and/or regions. In the United States, breast cancer incidence among women aged 50-84years rose over time and predominantly affected women aged 60 years and older since 1986 (Supplementary Figure 1A, left panel, available online). By contrast, in East Asian countries and/or regions, the incidence of breast cancer increased over time among women aged 40 years and older, becoming disproportionally higher in women aged 4075 years with concave downward or flat curves (Supplementary Figures 1, B-G, left panel, available online). Patterns of agespecific female breast cancer incidence over time were quite different in the United States and East Asia. Conversely, patterns of age-specific female colorectal cancer incidence over time in the United States and East Asia were rather similar (Supplementary Figures 1, A-G, right panel, available online).

The incidence of colorectal cancer among women aged 55 years and older increased monotonically with age in the United States and East Asia, but the index year from which this rise began varied among countries and/or regions; for example, the incidence in Taiwan has risen sharply since 1995 (Supplementary Figure 1B, right panel, available online). Figure 1, A and B, shows the observed age-specific incidence rates of female breast and colorectal cancers for each country and/or region for years 2011-2014, and Figure 1, C and D, their estimated age-specific incidence rates based on multiple linear regression models. Two multiple linear regression models contained only those covariates with non-zero regression coefficients (statistical significance reached in 31 of 90 comparisons and 26 of 90 comparisons for breast and colorectal cancers, respectively; the $P$ values was $\leq .01$, shown in Supplementary Table 1, available online) and fitted the observed data well (multivariable coefficient of determination $\left[R^{2}\right]=0.9919$ and 0.9975 , respectively). Figure $1, \mathrm{C}$ and $\mathrm{D}$, illustrates quite different patterns of age-specific female breast cancer incidence between the United States and East Asia, but similar patterns of agespecific female colorectal cancer incidence.

To verify racial differences, we plotted age-specific incidence rates of female breast and colorectal cancers for years 19902010 with US SEER data for C-J-K Asian Americans and nonHispanic white Americans (Supplementary Figure 1, H-J, available online). Unlike those of female colorectal cancer, concave downward curves of age-specific female breast cancer incidence were apparent in C-J-K Asian Americans and fell below those of non-Hispanic white Americans (Figure 1, E and F).

Trends in the Age-Specific Distributions of the Immunophenotypes and Histological Grades of Breast Cancer Between the United States and East Asia

The observed proportion of ER+ increased gradually with age in US women (Figure 2A), but the proportions of HER2+, TNBC, and histological grade III decreased with age (Figure 2, B-D). However, age associations of these immunophenotypes in hospital data from different East Asian countries and/or regions 
A Breast cancer, observed

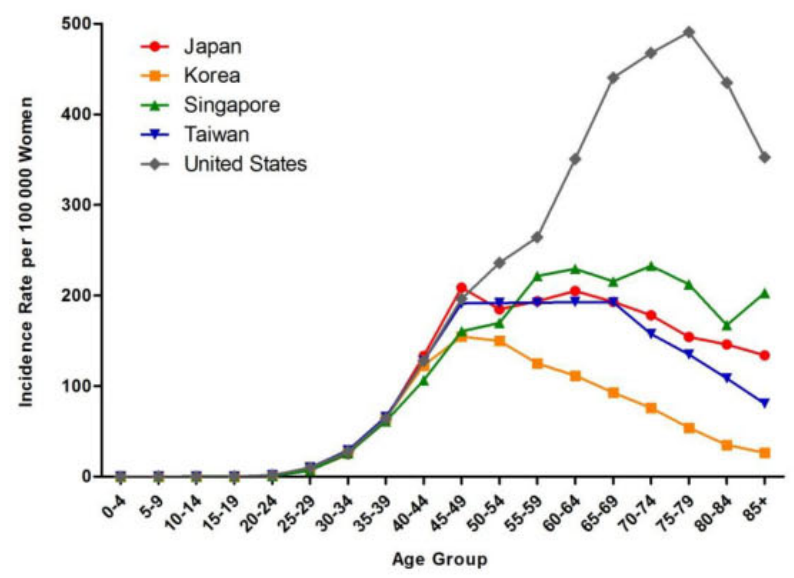

\section{Breast cancer, estimated}

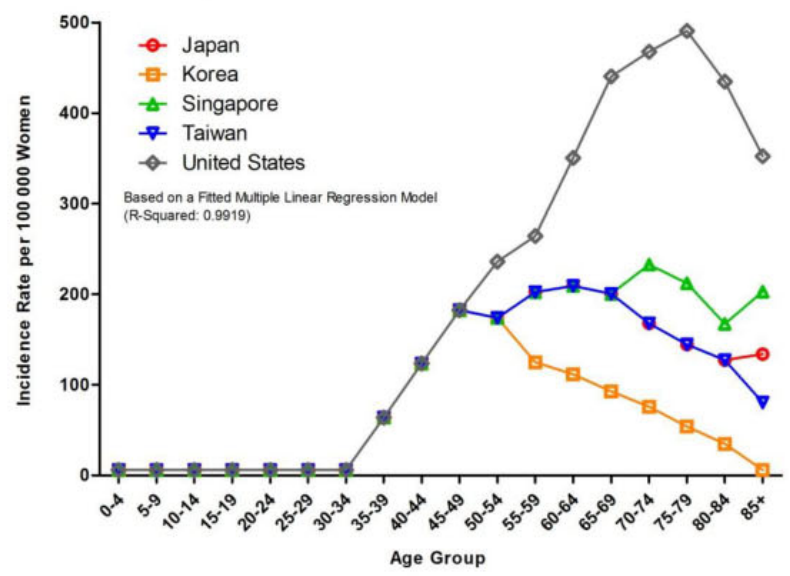

\section{E Breast cancer, observed}

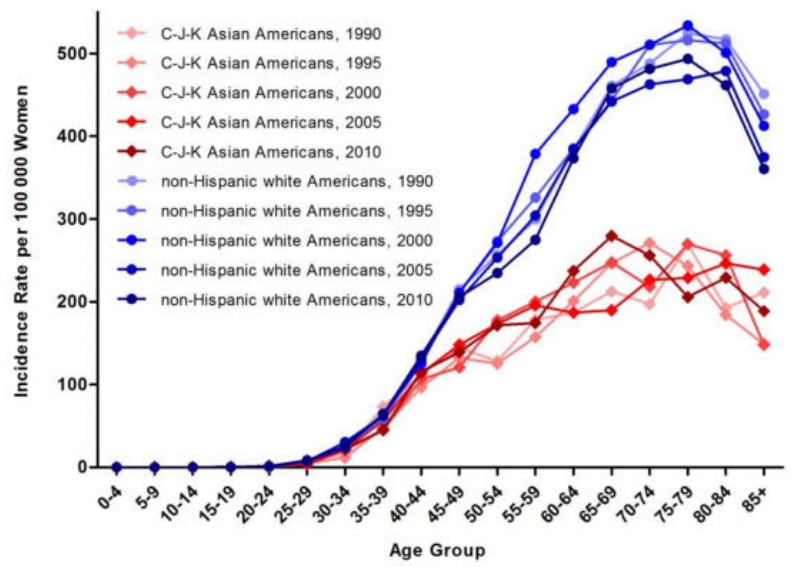

B Colorectal cancer, observed

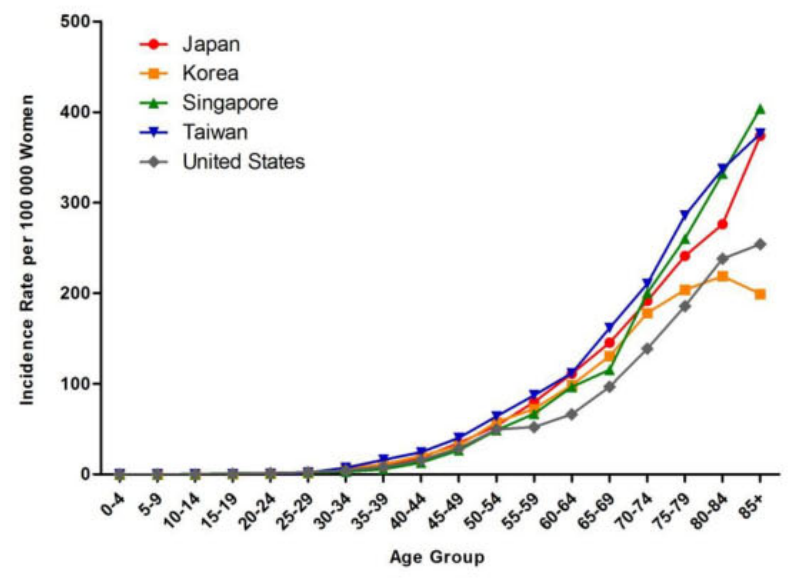

D Colorectal cancer, estimated

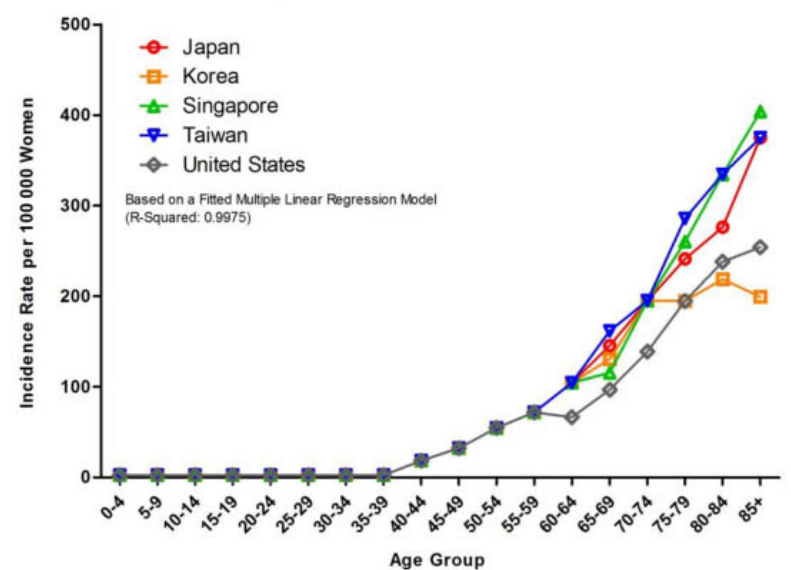

F Colorectal cancer, observed

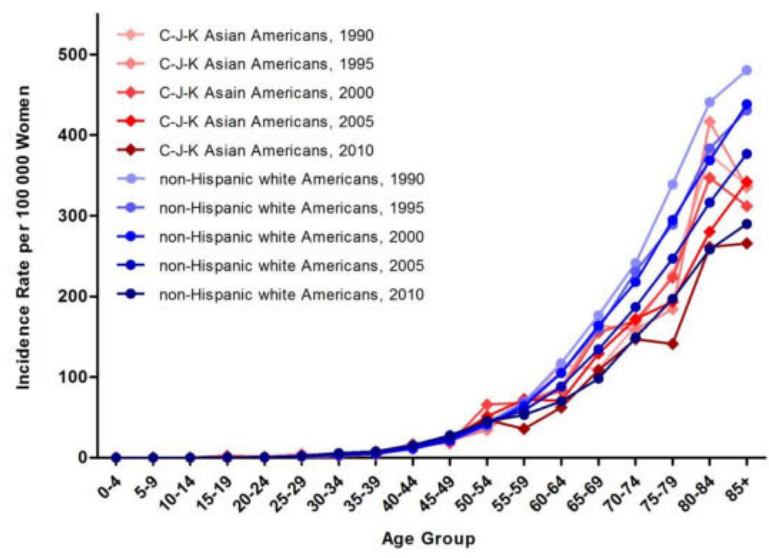

Figure 1. Age-specific incidence rates of female breast and colorectal cancers in East Asia and the United States and in SEER Asian Americans and non-Hispanic white Americans. A) The observed age-specific incidence rates of female breast cancer in East Asia $(n=253840)$ and the United States $(n=66230)$ for years $2011-2014$. B) The observed age-specific incidence rates of female colorectal cancer in East Asia $(n=184055)$ and the United States ( $n=17688)$ for years $2011-2014$. C) The estimated agespecific incidence rates of female breast cancer in East Asia and the United States for years 2011-2014. D) The estimated age-specific incidence rates of female colorectal cancer in East Asia and the United States for years 2011-2014. The estimated age-specific incidence rates shown in (C) and (D) were based on the fitted multiple linear regression models in Supplementary Table 1 (available online). $\mathrm{E}$ ) The observed age-specific incidence rates of female breast cancer in SEER Asian Americans ( $\mathrm{n}=$ $25665)$ and non-Hispanic white Americans ( $\mathrm{n}=683$ 164) for years 1990-2010. F) The observed age-specific incidence rates of female colorectal cancer in SEER Asian Americans $(n=11982)$ and non-Hispanic white Americans $(n=240286)$ for years 1990-2010. SEER = Surveillance, Epidemiology, and End Results. 
A $\mathrm{ER}+$, observed

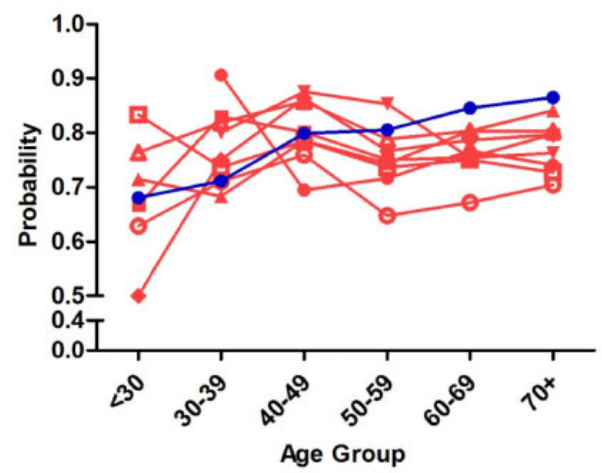

C TNBC, observed

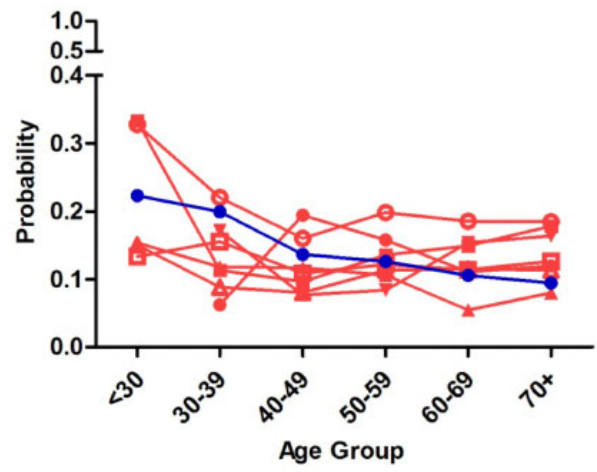

B HER2 +, observed

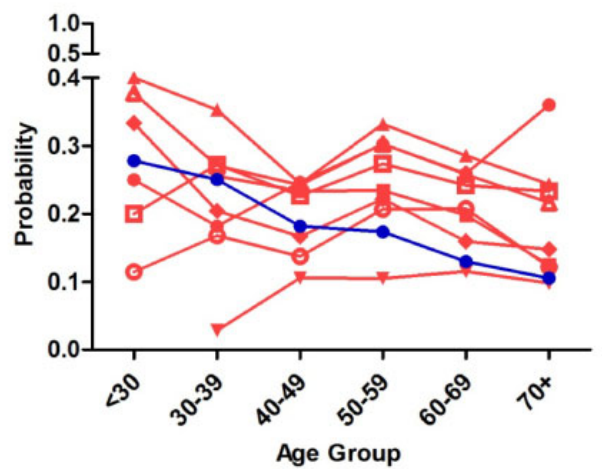

D Histological grade III, observed

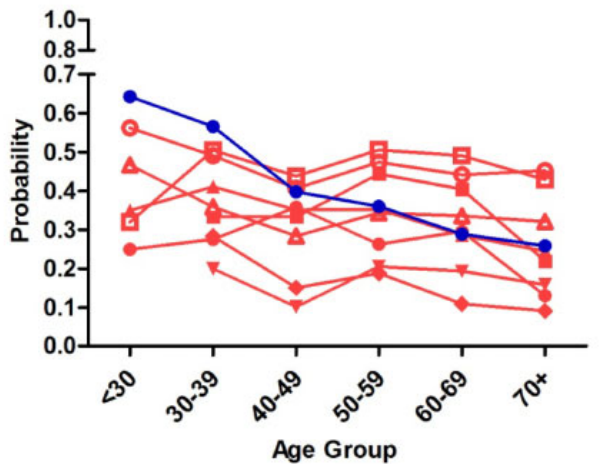

- $\mathrm{BCH}$, China, 2010-2012

- $\mathrm{PWH}$, Hong Kong, 2012

- WCD, Hong Kong, 2010-2014

$\rightarrow \mathrm{KUH}$, Japan, 2010-2012

$\neg$ NCCHE, Japan, 2010-2014

- SNUH, Korea, 2010-2014

\#- SGH/NCCS, Singapore, 2010-2014

- NTUH, Taiwan, 2010-2014

SEER18, US, 2010-2013

Figure 2. Observed age-specific probabilities of ER+ (A), HER2+ (B), TNBC (C), and histological grade III (D) female breast cancer in East Asia and the United States. Age-specific clinicopathological characteristics of female breast cancer were determined based on 18008 cases from hospital data in East Asian countries and/or regions and 244819 cases from US SEER data for years 2010-2014. BCH $=$ Beijing Cancer Hospital; ER $+=$ estrogen receptor positive; HER2 $+=$ human epidermal growth factor receptor 2 positive; KUH $=$ Kyorin University Hospital; NCCHE = National Cancer Center Hospital East; NCCS = National Cancer Centre Singapore; NTUH = National Taiwan University Hospital; PWH $=$ Prince of Wales Hospital; SGH = Singapore General Hospital; SNUH = Seoul National University Hospital; TNBC = triple-negative breast cancer; WCD = West Cluster Database.

were not uniform (Figure 2, A-D). To elucidate the effects of age and country and/or region, we performed logistic regression analyses for probabilities of ER+, HER2 + , TNBC, and histological grade III female breast cancer with US SEER data and pooled data from the eight East Asian hospitals over years 2010-2014 (Supplementary Table 2, available online); age groups were younger than 30, 30-39, 40-49, 50-59, 60-69, and 70years and older, and a dummy variable "Hospitals in East Asia" was specified $(1=$ hospital in East Asia, $0=$ United States). After adjusting for the unique effects of individual hospitals, the estimated probability of ER+ increased with age in the United States, but no similar trends were evident in East Asian patients: their probability of ER positivity at age 40-49years was statistically significantly higher (odds ratio $[\mathrm{OR}]=1.50,95 \%$ confidence interval $[\mathrm{CI}]=1.36$ to $1.67, \mathrm{P}<.001$ ), whereas the probability of ER positivity at age 50-59 years was statistically significantly lower ( $\mathrm{OR}=0.88,95 \% \mathrm{CI}=0.82$ to $0.95, \mathrm{P}<.001$ ). The estimated probability of TNBC decreased with age in the United States, but not in East Asian patients; their probability of TNBC positivity at age 40-49 years was $0.79(95 \% \mathrm{CI}=0.71$ to $0.88, \mathrm{P}<.001)$ (Supplementary Table 2, available online).

To visualize effect patterns, we made conditional effect plots of estimated age-specific probabilities of ER+, HER2+, TNBC, and histological grade III subtypes of female breast cancer for years 2010-2014 for the United States and East Asia, based on our fitted multiple logistic regression models (Supplementary Table 2, available online). Associations of these markers with age were quite different between American and East Asian women with breast cancer (Figure 3). As shown in Supplementary Table 2 (available online), the estimated agespecific probability of ER+ increased statistically significantly with age in US women (all $P$ values associated with age $\leq .01$ ), whereas those of HER2+, TNBC, and histological grade III breast cancer declined statistically significantly with age (all $P$ values associated with age $\leq 0.005$ ). In contrast, the estimated agespecific probability of ER+ in East Asian women with breast cancer peaked at age 40-49years, HER2+ at 50-59years, TNBC at all ages except 40-49years, and histological grade III below age 30 years. We also applied this approach to analyzing the observed age-specific proportions of PR+, HR+/HER2-, HR+/ HER2+, and HR-/HER2 + subtypes of female breast cancer in the United States and the hospitals in East Asia (Supplementary Figures 2 and 3, available online).

\section{Distributions of Age-Specific Pathological \\ Characteristics Between Asian and White Americans}

To verify racial differences, we analyzed the distributions of age-specific pathological characteristics between Asian and white Americans. Intriguingly, the differences between the curves plotted for age-specific probabilities of ER+, HER2+, and TNBC in C-J-K Asian Americans and white Americans (Figure 4, A-C) resembled those observed between East Asian and US women (Figure 3, A-C). The histological grade III tumors 
A $\quad \mathrm{ER}+$, estimated

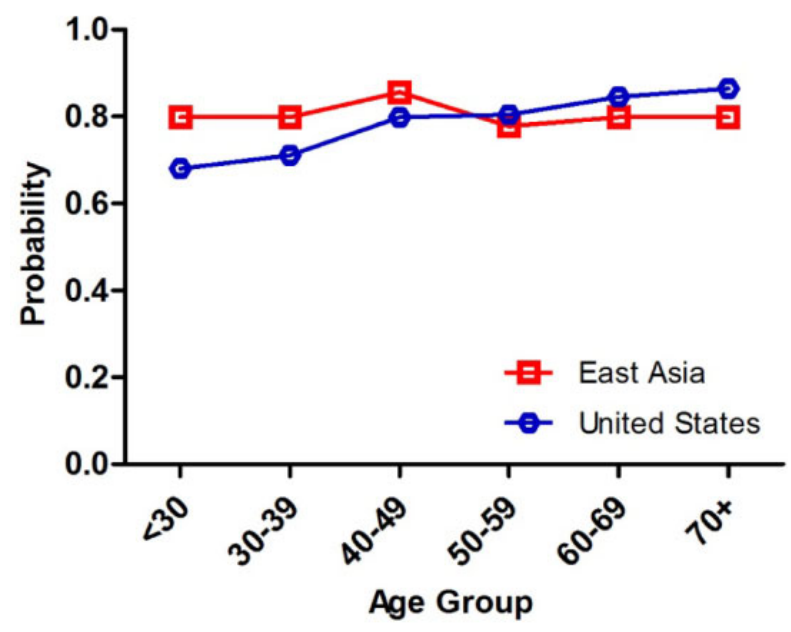

C TNBC, estimated

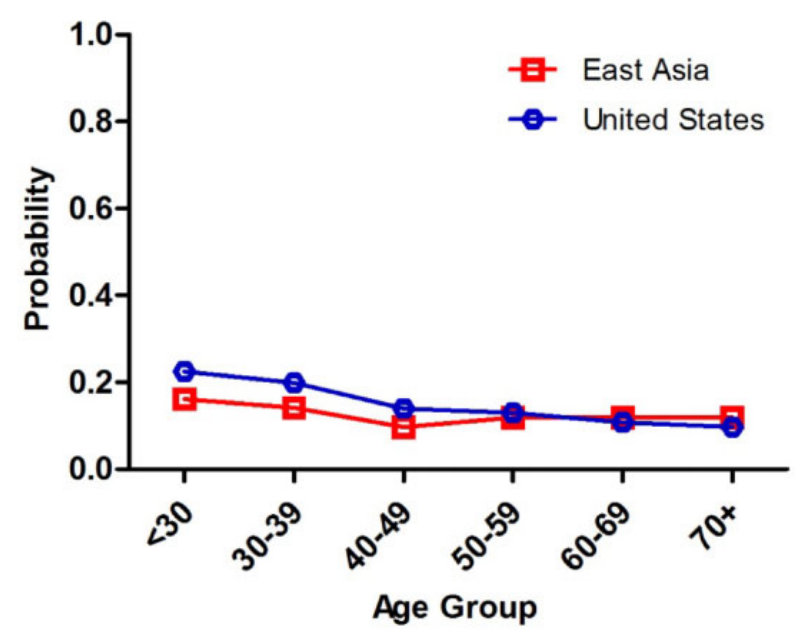

B HER2 +, estimated

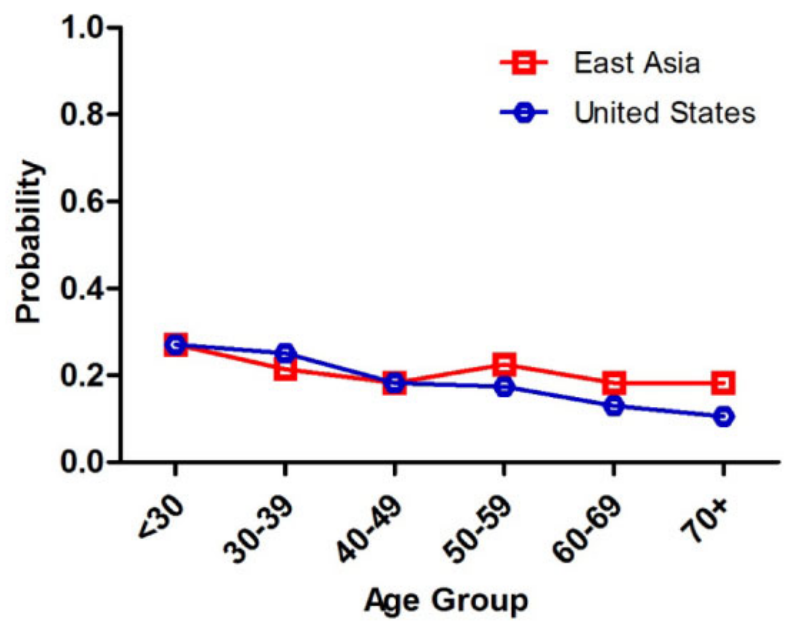

D Histological grade III, estimated

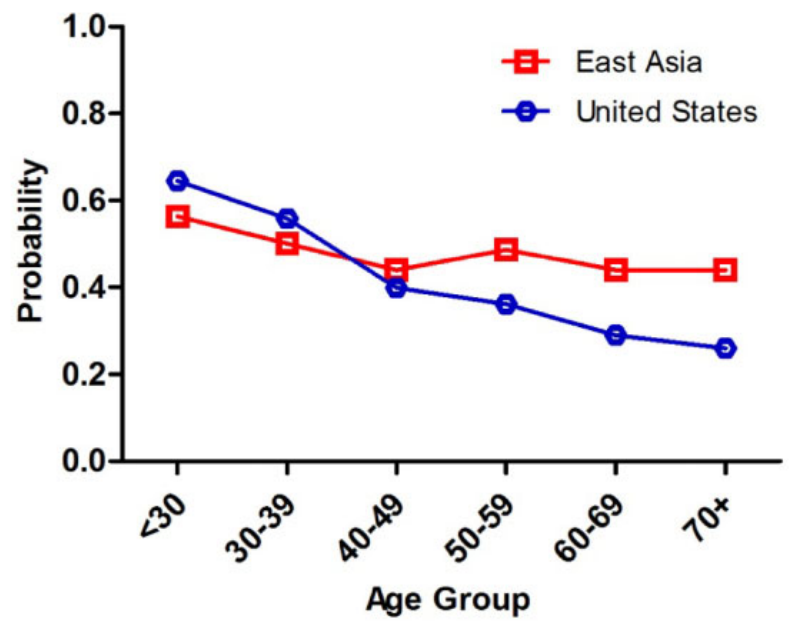

Figure 3. Conditional effect plots of estimated age-specific probabilities of ER+ (A), HER2+ (B), TNBC (C), and histological grade III (D) female breast cancer in East Asia and the United States for years 2010-2014. The estimated age-specific clinicopathological characteristics of female breast cancer were determined based on the fitted multiple logistic regression models of 18008 cases in East Asians and 244819 in Americans (Supplementary Table 2, available online). ER+ = estrogen receptor positive; HER2 + = human epidermal growth factor receptor 2 positive; TNBC = triple-negative breast cancer.

in C-J-K Asian Americans were more like those of white Americans (Figure 4D) and different from those of East Asians (Figure 3D). In addition, the differences between the curves plotted for age-specific probabilities of PR+, HR+/HER2-, and HR-/ HER2 + in C-J-K Asian Americans and white Americans (Supplementary Figure 4, A, B, and D, available online) resembled those observed between East Asian and US women (Supplementary Figure 3, A, B, and D, available online). The $\mathrm{HR}+/ \mathrm{HER} 2+$ tumors in $\mathrm{C}-\mathrm{J}-\mathrm{K}$ Asian Americans were more like those of white Americans (Supplementary Figure 4C, available online) and different from those of East Asians (Supplementary Figure 3C, available online).

\section{Discussion}

This study identified age-specific pathological differences in breast cancer between Asians and non-Asians that may signify racial differences in its etiology and biology.
During the past four decades, the incidences of breast cancer and colorectal cancer in East Asian women have increased rapidly; the etiologies of both malignancies relate to diet and lifestyle (eg, physical inactivity, smoking, and drinking alcohol) $(9,10)$. Consequently, the profile of increasing age-specific incidence of colorectal cancer in East Asian women closely resembled that in US women in recent years, suggesting that Westernization during more than two decades in East Asia has indeed increased women's risk of colorectal cancer. Variation between East Asian countries and/or regions in when the agespecific incidence of female colorectal cancer began to rise probably reflects differing speeds of Westernization. However, markedly increasing breast cancer incidence in relatively younger East Asian women cannot be attributed solely to the effect of Westernization.

Different distributions in age-specific pathological characteristics of female breast cancer between major hospitals in East Asian countries and/or regions and the United States 
A ER +, observed

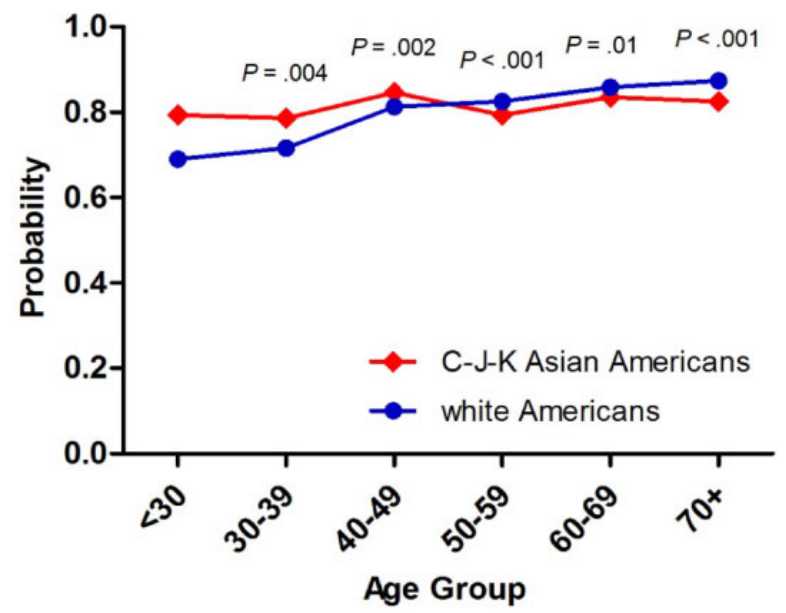

C TNBC, observed

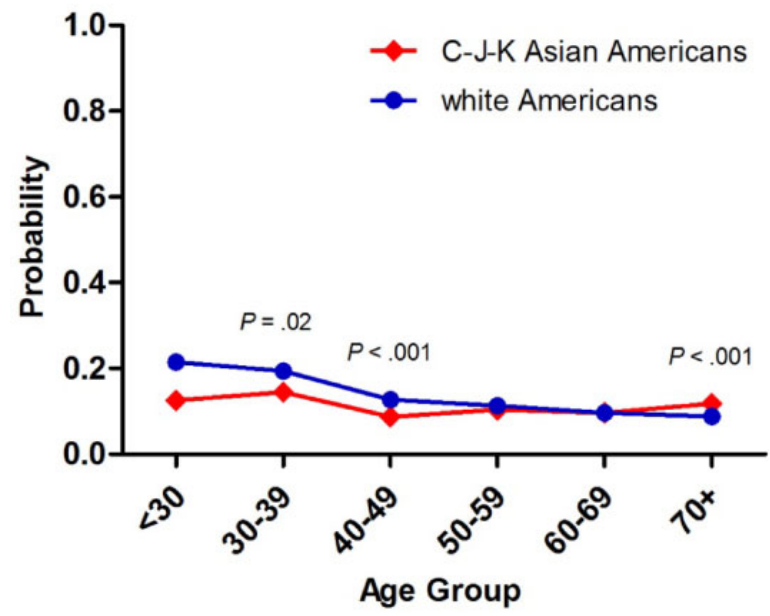

B HER2 +, observed

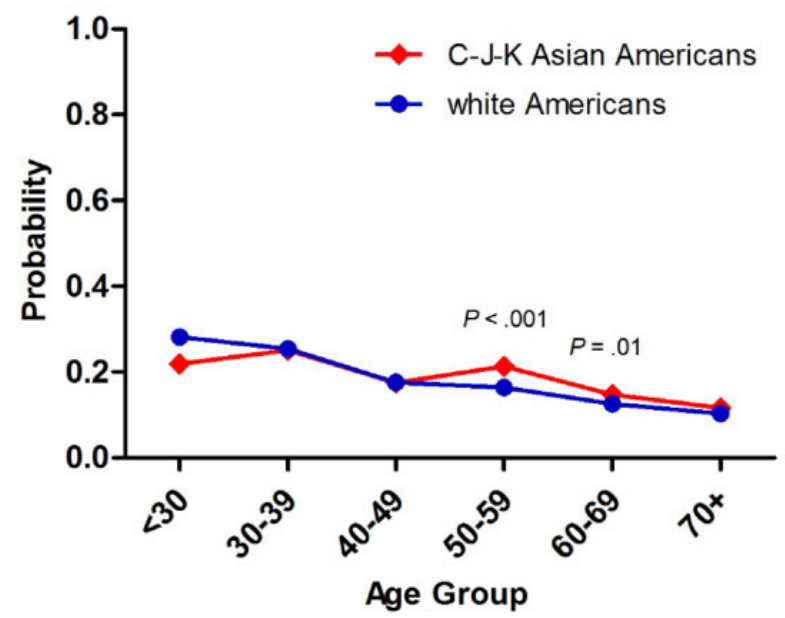

D Histological grade III, observed

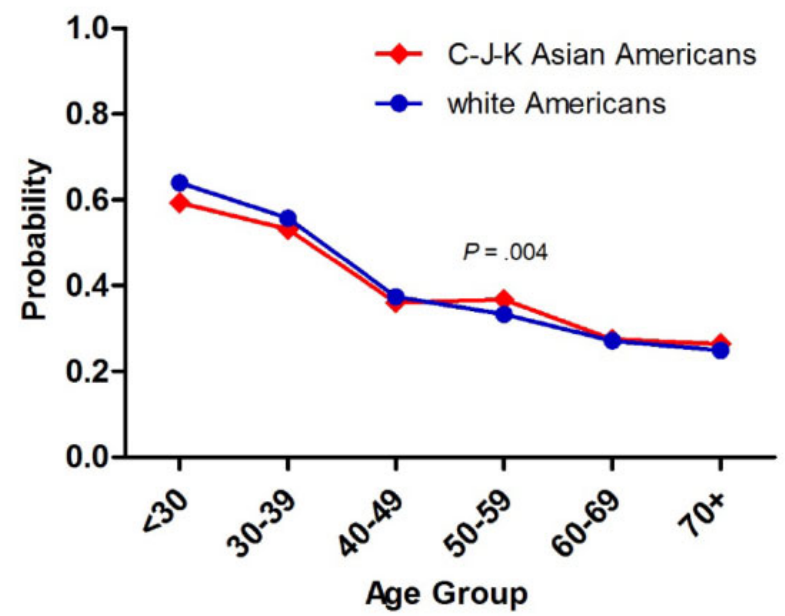

Figure 4. Observed age-specific proportions of ER+ (A), HER2+ (B), TNBC (C), and histological grade III (D) female breast cancer in Surveillance, Epidemiology, and End Results program Asian Americans and white Americans for years 2010-2013. The age-specific proportions of ER+, HER2+, TNBC, and histological grade III between 7185 C-J-K Asian Americans and 194524 white Americans were compared using two-sided pointwise two-sample $z$ tests. The figures show the exact P values when $P<$ .05. C-J-K = Chinese-Japanese-Korean; ER $+=$ estrogen receptor positive; HER2 $+=$ human epidermal growth factor receptor 2 positive; TNBC $=$ triple-negative breast cancer.

revealed a racial effect on the etiology of female breast cancer. Moreover, comparisons in age-specific incidence and pathological characteristics of female breast cancer between C-J-K Asian Americans and white Americans discriminated the effects of Westernized lifestyle and racial ancestry.

It is difficult to disentangle the intertwined genetic and environmental influences without knowing how long the Asian American immigrants had lived in the United States. Nevertheless, high probabilities of ER+ breast cancer in younger East Asian women and C-J-K Asian Americans imply that estrogen plays an important etiologic role in younger Asian women. Moreover, the higher probabilities of $\mathrm{ER}+$ and $\mathrm{PR}+$ and lower probability of TNBC in East Asian women who are younger than 50 years compared with American women are consistent with previous studies. In Taiwan and Japan, women aged younger than 50 years had higher probabilities of $\mathrm{ER}+$ or $\mathrm{PR}+$ and lower probability of TNBC than older women (29-31). Relatively high prevalences of ER+ and $\mathrm{PR}+$ in Asian women aged 4049 years indicate an estrogen-related causality, as the incidences of endometrioid carcinomas of the uterus and ovary have also increased rapidly in younger East Asian women $(7,32,33)$. The latter cancer subtypes have highly prevalent hormone receptor expressions and are etiologically linked to estrogen exposure.

On the other hand, Asian women born after the 1960s have higher caloric and animal-based protein intake than ancestral generations, which may increase endogenous estrogen production and lead to earlier menarche (34). Early studies conducted among premenopausal women in Japan (35), China $(36,37)$, and recent Southeast Asian immigrants to Hawaii (38) reported serum estradiol levels 30\%-70\% lower in Asians than white contemporaries. Yet, an immigration study published 
approximately 10 years later found comparable plasma estradiol levels between Asian and Western premenopausal women (39), and another study even showed a higher estradiol level in Asian Americans than Caucasians (40). In addition to earlier men-arche, drastically reduced fertility rates and delayed childbearing in East Asian countries $(41,42)$ could increase women's risk of breast cancer. Exogenous estrogen exposure by industrial and environmental estrogenic pollutants is another potential cause of breast cancer. The rapid industrialization during the 1960s in East Asia led to more exposures to cosmetic products, pesticides, and plastics that contained estrogenic ingredients during manufacturing or produced estrogenic pollutants after degradation (43-46). Such changes of lifestyle, society, and environments in East Asia could interact with genetic predisposition to produce a unique profile of age-specific female breast cancer incidence. Direct comparisons of these potential risk factors and genetic predispositions between East Asian and European ancestry are warranted.

Although expressions of certain immunophenotypic markers were similar between C-J-K Asian Americans and East Asians, the age-specific probabilities of $\mathrm{HR}+/ \mathrm{HER} 2+$ and histological grade III tumors in C-J-K Asian Americans were more like those of white Americans and different from East Asians. Thus, the differences between East Asians and C-J-K Asian Americans in lifestyle and environmental exposures might also affect the clinicopathological characteristics and risk of breast cancer. For example, the consumption of green tea and soy products differs between East Asians and C-J-K Asian Americans. Some studies found that that green tea and soy isoflavone consumption among Asian women reduced their risks of breast cancer and recurrence (47-49).

Finally, we acknowledge that this study has some limitations. First, national registry data covering certain time periods in some East Asian countries and/or regions were not available. Second, data from the participating hospitals in East Asia might not be nationally representative. However, this might not be a serious concern because one of the major findings concerning the unique age-specific probabilities of $\mathrm{ER}+$ in East Asia evident in the hospital databases evaluated in this study was consistent with other studies that assessed the national registries in Taiwan (30) and Japan (31). Third, as in a previous study using the US SEER data (50), the criteria for ER and PR positivity could differ somewhat between participating hospitals. Nonetheless, the frequencies of low (1\%-10\%) ER and PR tumors were $4.3 \%$ and $12.8 \%$, respectively, and the distributions of age-specific probabilities of ER and PR positivity were similar whether using $1 \%$ or $10 \%$ as the cutoff in this study (data not shown). In addition, the distributions were established separately in East Asian and US populations. Last, different cancer screening policies and accessibility of screening tools in East Asian countries and/or regions and the United States might affect the age-specific incidence rates of female breast cancer and colorectal cancer.

In summary, the proportions of key pathological features of breast cancer across different age groups in East Asian women were statistically significantly different from those of white American women but resembled those of C-J-K Asian Americans. Unlike colorectal cancer, lifestyle Westernization cannot fully account for the rapidly increasing incidence of breast cancer in younger East Asian women. Interactions of race-associated genetic and environmental factors might exacerbate carcinogenesis in younger East Asian women with estrogen-related malignancies.

\section{Funding}

This research initiative was conceived and discussed during the StAR (Strategy in the Asia Region) Premenopausal Breast Cancer Expert Forums, supported by Novartis Asia Pacific region (including Novartis Taiwan and other countries). The Asian Breast Cancer Cooperative Group was inaugurated during the 3rd StAR Premenopausal Breast Cancer Expert Forum.

\section{Notes}

Affiliations of authors: National Taiwan University Hospital, Taipei, Taiwan (CHL, SMH, CSH, ALC, YSL); National Cancer Centre Singapore, Singapore (YSY, BT); Seoul National University Hospital, Cancer Research Institute, Seoul National University College of Medicine, Seoul, Republic of Korea (KHL, SAI, WH); National Cancer Center Hospital East, Chiba, Japan (YN); Chinese University of Hong Kong, Hong Kong, China (WY); Cancer Institute Hospital, Tokyo, Japan (TU); The University of Hong Kong, Hong Kong, China (AK, RL); Peking University Cancer Hospital, Beijing, China (HL); SingHealth Duke NUS Breast Centre, Sengkang General Hospital, Singapore General Hospital, Singapore (BT); Graduate Institute of Clinical Medicine and School of Nursing, College of Medicine, National Taiwan University, Taipei, Taiwan (FCH); Statistical Consulting Clinic, International-Harvard Statistical Consulting Company, Taipei, Taiwan (FCH).

Novartis Asia Pacific region and countries had no role in the design and conduct of the research; collecting, managing, analyzing, or interpreting data; or preparing the manuscript; nor the decision to submit the manuscript for publication. None of the authors have conflicts of interest to report.

Author contributions: Yen-Shen Lu had full access to all the data in the study and takes responsibility for the integrity of the data and the accuracy of the data analysis. Study concept and design: Ching-Hung Lin, Seock-Ah Im, and Yen-Shen Lu. Acquisition, analysis, or interpretation of data: all authors. Drafting of the manuscript: Ching-Hung Lin, Fu-Chang Hu, and Yen-Shen Lu. Critical revision of the manuscript for important intellectual content: all authors. Statistical analysis: Fu-Chang Hu.

Additional contributions: We thank database manager Jung Youn Kim and colleagues Tae-Yong Kim, MD; Han-Byoel Lee, MD; Hyeong-Gon Moon, MD; In Ae Park, MD, PhD; and DongYoung Noh, MD, PhD, Seoul National University Hospital; Yaxin Liu, MD, Beijing Cancer Hospital; and Shigeru Imoto, MD, PhD, Kyorin University Hospital, for contributing to patient data collection. We would also like to acknowledge the contributions of the National Registry of Disease Office of Singapore and colleagues who contributed to the Singapore Health Services Joint Breast Cancer Registry, including Fuh-Yong Wong, MD, and Kong-Wee Ong, MD, PhD. We thank David Neil, PhD, of ContentEd Net (Taiwan) and Full Universe Integrated Marketing Ltd (Taiwan), for professional editorial services, which were funded by National Taiwan University Hospital.

\section{References}

1. Bray F, McCarron P, Parkin DM. The changing global patterns of female breast cancer incidence and mortality. Breast Cancer Res. 2004;6(6):229-239.

2. Shin HR, Joubert C, Boniol M, et al. Recent trends and patterns in breast cancer incidence among Eastern and Southeastern Asian women. Cancer Causes Control. 2010;21(11):1777-1785.

3. Youlden DR, Cramb SM, Yip CH, Baade PD. Incidence and mortality of female breast cancer in the Asia-Pacific region. Cancer Biol Med. 2014;11(2):101-115. 
4. Minami Y, Tsubono Y, Nishino Y, Ohuchi N, Shibuya D, Hisamichi S. The increase of female breast cancer incidence in Japan: emergence of birth cohort effect. Int J Cancer. 2004;108(6):901-906.

5. Shen YC, Chang CJ, Hsu C, Cheng CC, Chiu CF, Cheng AL. Significant difference in the trends of female breast cancer incidence between Taiwanese and Caucasian Americans: implications from age-period-cohort analysis. Cancer Epidemiol Biomarkers Prev. 2005;14(8):1986-1990.

6. Matsuno RK, Anderson WF, Yamamoto S, et al. Early- and late-onset breast cancer types among women in the United States and Japan. Cancer Epidemiol Biomarkers Prev. 2007;16(7):1437-1442.

7. Lin $\mathrm{CH}$, Chen YC, Chiang CJ, et al. The emerging epidemic of estrogen-related cancers in young women in a developing Asian country. Int J Cancer. 2012; 130(11):2629-2637.

8. Sung H, Rosenberg PS, Chen WQ et al. Female breast cancer incidence among Asian and Western populations: more similar than expected. J Natl Cancer Inst. 2015;107(7): djv107

9. Porter P. "Westernizing" women's risks? Breast cancer in lower-income countries. N Engl J Med. 2008;358(3):213-216.

10. Wynder EL, Fujita Y, Harris RE, Hirayama T, Hiyama T. Comparative epidemiology of cancer between the United States and Japan. A second look. Cancer. 1991;67(3):746-763.

11. Amadou A, Ferrari P, Muwonge R, et al. Overweight, obesity and risk of premenopausal breast cancer according to ethnicity: a systematic review and dose-response meta-analysis. Obes Rev. 2013;14(8):665-678.

12. Wada K, Nagata C, Tamakoshi A, et al. Body mass index and breast cancer risk in Japan: a pooled analysis of eight population-based cohort studies. Ann Oncol. 2014;25(2):519-524.

13. Wu AH, Yu MC, Tseng CC, Pike MC. Epidemiology of soy exposures and breast cancer risk. Br J Cancer. 2008;98(1):9-14

14. Hartman M, Suo C, Lim WY, Miao H, Teo YY, Chia KS. Ability to predict breast cancer in Asian women using a polygenic susceptibility model. Breast Cancer Res Treat. 2011;127(3):805-812.

15. Kan Z, Ding Y, Kim J, et al. Multi-omics profiling of younger Asian breast can cers reveals distinctive molecular signatures. Nat Commun. 2018;9(1):1725.

16. Arnold M, Sierra MS, Laversanne M, et al. Global patterns and trends in colorectal cancer incidence and mortality. Gut. 2017;66(4):683-691.

17. Bray F, Ferlay J, Soerjomataram I, et al. Global cancer statistics 2018 : GLOBOCAN estimates of incidence and mortality worldwide for 36 cancers in 185 countries. CA Cancer J Clin. 2018;68(6):394-424.

18. Cancer Information Service NCC, Japan. National estimates of cancer incidence based on cancer registries in Japan (1975-2013). http://ganjoho.jp/en/ professional/statistics/table_download.html. Accessed January 12, 2017.

19. Oh CM, Won YJ, Jung KW, et al. Cancer statistics in Korea: incidence, mortality, survival, and prevalence in 2013. Cancer Res Treat. 2016;48(2):436-450.

20. Lee CM, Zheng H, Tan VK, et al. Surgery for early breast cancer in the extremely elderly leads to improved outcomes-An Asian population study Breast. 2017;36:44-48.

21. System NHSCROI. Health Promotion Administration, Ministry of Health and Welfare, Taiwan (1979-2013).https://cris.hpa.gov.tw/pagepub/Home.aspx. Accessed September 2, 2016.

22. Organization IAfRoCWH. CI5plus, cancer incidence in five continents time trends. http://ci5.iarc.fr/CI5plus/Pages/online.aspx. Accessed December 29, 2016.

23. United States National Cancer Institute Surveillance, Epidemiology, and End Results (SEER) Program. SEER*Stat Database: Incidence-SEER 9 Regs Research Data. www.seer.cancer.gov. Accessed November 8, 2016.

24. United States National Cancer Institute, Surveillance, Epidemiology, and End Results (SEER) Program. SEER*Stat Database: Incidence-SEER 18 Regs Research Data. www.seer.cancer.gov. Accessed December 16, 2016.

25. Hammond ME, Hayes DF, Dowsett M, et al. American Society of Clinical Oncology/College of American Pathologists guideline recommendations for immunohistochemical testing of estrogen and progesterone receptors in breast cancer. Arch Pathol Lab Med. 2010;134(6):907-922.

26. Ogawa $\mathrm{YM}$, Kato $\mathrm{Y}$, Oguma $\mathrm{M}$, et al. Immunohistochemical assessment for estrogen receptor and progesterone receptor status in breast cancer: analysis for a cut-off point as the predictor for endocrine therapy. Breast Cancer. 2004; 11(3):267-275

27. Prat A, Cheang MC, Martin M, et al. Prognostic significance of progesterone receptor-positive tumor cells within immunohistochemically defined luminal A breast cancer. J Clin Oncol. 2013;31(2):203-209.
28. United States National Cancer Institute, Surveillance, Epidemiology, and End Results (SEER) Program. SEER*Stat Database: Incidence-SEER 11. www.seer. cancer.gov. Accessed January 25, 2017.

29. Lin CH, Liau JY, Lu YS, et al. Molecular subtypes of breast cancer emerging in young women in Taiwan: evidence for more than just westernization as a reason for the disease in Asia. Cancer. Epidemiol Biomarkers Prev. 2009;18(6): 1807-1814

30. Lin $\mathrm{CH}$, Chuang PY, Chiang CJ, et al. Distinct clinicopathological features and prognosis of emerging young-female breast cancer in an East Asian country: a nationwide cancer registry-based study. Oncologist. 2014;19(6): 583-591.

31. Kurebayashi J, Miyoshi Y, Ishikawa T, et al. Clinicopathological characteristics of breast cancer and trends in the management of breast cancer patients in Japan: based on the Breast Cancer Registry of the Japanese Breast Cancer Society between 2004 and 2011. Breast Cancer. 2015;22(3):235-244.

32. Ushijima K. Current status of gynecologic cancer in Japan. J Gynecol Oncol. 2009;20(2):67-71.

33. Lee JY, Kim EY, Jung KW, et al. Trends in gynecologic cancer mortality in East Asian regions. J Gynecol Oncol. 2014;25(3):174-182.

34. Sans P, Combris P. World meat consumption patterns: an overview of the last fifty years (1961-2011). Meat Sci. 2015;109:106-111.

35. MacMahon B, Cole P, Brown JB, et al. Oestrogen profiles of Asian and North American women. Lancet. 1971;2(7730):900-902.

36. Bernstein L, Yuan JM, Ross RK, et al. Serum hormone levels in premenopausal Chinese women in Shanghai and white women in Los Angeles: results from two breast cancer case-control studies. Cancer Causes Control. 1990;1(1):51-58.

37. Key TJ, Chen J, Wang DY, Pike MC, Boreham J. Sex hormones in women in rural China and in Britain. Br J Cancer. 1990;62(4):631-636.

38. Goldin BR, Adlercreutz H, Gorbach SL, et al. The relationship between estrogen levels and diets of Caucasian American and Oriental immigrant women Am J Clin Nutr. 1986;44(6):945-953.

39. Falk RT, Fears TR, Hoover RN, et al. Does place of birth influence endogenous hormone levels in Asian-American women? Br J Cancer. 2002;87(1):54-60.

40. Pinheiro SP, Holmes MD, Pollak MN, Barbieri RL, Hankinson SE. Racial differences in premenopausal endogenous hormones. Cancer Epidemiol Biomarkers Prev. 2005;14(9):2147-2153.

41. Silva T, Tenreyro S. Population control policies and fertility convergence. $J$ Econ Perspect. 2017;31(4):205-228.

42. United Nations, Department of Economic and Social Affairs, Population Division (2017). World Population Prospects: The 2017 Revision, Volume II: Demographic Profiles. ST/ESA/SER.A/400. https://esa.un.org/unpd/wpp/ Publications/. Accessed December 12, 2017.

43. Lu YY, Chen ML, Sung FC, Wang PS, Mao IF. Daily intake of 4-nonylphenol in Taiwanese. Environ Int. 2007;33(7):903-910.

44. Chen CY, Wen TY, Wang GS, Cheng HW, Lin YH, Lien GW. Determining estrogenic steroids in Taipei waters and removal in drinking water treatment using high-flow solid-phase extraction and liquid chromatography/tandem mass spectrometry. Sci Total Environ. 2007;378(3):352-365.

45. Shao B, Hu J, Yang M, An W, Tao S. Nonylphenol and nonylphenol ethoxylates in river water, drinking water, and fish tissues in the area of Chongqing, China. Arch Environ Contam Toxicol. 2005;48(4):467-473.

46. Li B, Liu R, Gao H, Tan R, Zeng P, Song Y. Spatial distribution and ecological risk assessment of phthalic acid esters and phenols in surface sediment from urban rivers in Northeast China. Environ Pollut. 2016;219: 409-415.

47. Nakachi K, Suemasu K, Suga K, Takeo T, Imai K, Higashi Y. Influence of drinking green tea on breast cancer malignancy among Japanese patients. Jpn J Cancer Res. 1998;89(3):254-261.

48. Gao Y, Huang YB, Liu XO, et al. Tea consumption, alcohol drinking and physical activity associations with breast cancer risk among Chinese females: a systematic review and meta-analysis. Asian Pac J Cancer Prev. 2013;14(12): 7543-7550.

49. Dong JY, Qin LQ. Soy isoflavones consumption and risk of breast cancer incidence or recurrence: a meta-analysis of prospective studies. Breast Cancer Res Treat. 2011;125(2):315-323.

50. Howlader N, Altekruse SF, Li CI, et al. US incidence of breast cancer subtypes defined by joint hormone receptor and HER2 status. J Natl Cancer Inst. 2014; 106(5): dju055. 